\title{
Serious Adverse Drug Reactions in Children and Adolescents Treated On- and Off-Label with Antidepressants and Antipsychotics in Clinical Practice
}

(ㅇ)( $\odot \ominus$

Authors

Karin M. Egberts ${ }^{1}$, Manfred Gerlach¹, Christoph U. Correll2, 3, 4, Paul L. Plener 5, 6, Uwe Malzahn7, Peter Heuschmann7, 8 , Stefan Unterecker ${ }^{9}$, Maike Scherf-Clavel ${ }^{9}$, Hans Rock ${ }^{10}$, Gisela Antony ${ }^{10}$, Wolfgang Briegel ${ }^{1,}{ }^{11}$, Christian Fleischhaker ${ }^{12}$, Alexander Häge ${ }^{13}$, Tobias Hellenschmidt ${ }^{14}$, Harmut Imgart ${ }^{15}$, Michael Kaess ${ }^{16,}{ }^{17}$, Andreas Karwautz ${ }^{6}$, Michael Kölch ${ }^{18,19}$, Karl Reitzle 20, Tobias Renner ${ }^{21}$, Su-Yin Reuter-Dang1, 20, Christian Rexroth ${ }^{22}$, Gerd Schulte-Körne ${ }^{23}$, Frank M. Theisen ${ }^{24}$, Susanne Walitza ${ }^{25}$, Christoph Wewetzer ${ }^{26}$, Stefanie Fekete', Regina Taurines' ${ }^{1}$, Marcel Romanos ${ }^{1}$

Affiliations

1 Department of Child and Adolescent Psychiatry, Psychosomatics and Psychotherapy, Center of Mental Health, University Hospital Wuerzburg, Wuerzburg, Germany

2 Department of Child and Adolescent Psychiatry, Charité Universitätsmedizin Berlin, Berlin, Germany

3 The Zucker Hillside Hospital, Department of Psychiatry, Northwell Health, Glen Oaks, NY, USA

4 Donald and Barbara Zucker School of Medicine at Hofstra/Northwell, Department of Psychiatry and Molecular Medicine, Hempstead, NY, USA

5 Department of Child and Adolescent Psychiatry/ Psychotherapy, University Hospital Ulm, Ulm, Germany

6 Department of Child and Adolescent Psychiatry, Medical University Vienna, Vienna, Austria

7 Clinical Trial Center Wuerzburg, University Hospital Wuerzburg, Wuerzburg, Germany

8 Institute of Clinical Epidemiology and Biometry, University of Wuerzburg, Germany

9 Department of Psychiatry, Psychosomatics and Psychotherapy, Center of Mental Health, University Hospital Wuerzburg, Wuerzburg, Germany

10 Central Information Office, Department of Neurology, Philipps University of Marburg, Marburg, Germany

11 Department of Child and Adolescent Psychiatry, Psychosomatics and Psychotherapy, Leopoldina Hospital, Schweinfurt, Germany

12 Department of Child and Adolescent Psychiatry and Psychotherapy, University Medical Center Freiburg, Freiburg, Germany

13 Department of Child and Adolescent Psychiatry and Psychotherapy, Central Institute of Mental Health, Medical Faculty Mannheim, Heidelberg University, Mannheim, Germany

14 Department of Child and Adolescent Psychiatry, Psychotherapy and Psychosomatic Medicine, Vivantes Clinic Berlin Neukölln, Berlin, Germany
15 Parkland-Clinic, Clinic for Psychosomatics and Psychotherapy, Academic Teaching hospital for the University Gießen, Bad Wildungen, Germany

16 Clinic for Child and Adolescent Psychiatry, Center for Psychosocial Medicine, University Hospital Heidelberg, Heidelberg, Germany

17 University Hospital of Child and Adolescent Psychiatry and Psychotherapy, University of Bern, Switzerland

18 Department of Child and Adolescent Psychiatry and Psychotherapy, Brandenburg Medical School Brandenburg, Neuruppin, Germany

19 Department of Child and Adolescent Psychiatry, Neurology, Psychosomatics, and Psychotherapy, University Medical Center Rostock, Rostock, Germany

20 Specialist practice and Medical Care Center for Child and Adolescent Psychiatry Munich, Munich, Germany

21 Department of Child and Adolescent Psychiatry, Psychosomatics and Psychotherapy, University Hospital of Psychiatry and Psychotherapy Tuebingen, Center of Mental Health Tuebingen, Germany

22 Clinic for Child and Adolescent Psychiatry, Psychosomatics and Psychotherapy at the Regensburg District Hospital, Medbo KU, University Hospital, Regensburg, Germany

23 Department of Child and Adolescent Psychiatry, Psychosomatics and Psychotherapy, LudwigMaximilians-University (LMU) Hospital, Munich, Germany

24 Herz-Jesu-Krankenhaus gGmbH, Department of Child and Adolescent Psychiatry and Psychotherapy, Fulda, Germany

25 Department of Child and Adolescent Psychiatry and Psychotherapy, University Hospital of Psychiatry Zurich, Switzerland

26 Kliniken der Stadt Köln gGmbH, Clinic for Child and Adolescent Psychiatry Holweide, Children's Hospital Amsterdamer Straße, Cologne, Germany 
Key words

antidepressants, antipsychotics, child, adverse drug reactions, safety

received 09.09 .2021

revised 02.11 .2021

accepted 29.11.2021

published online $\quad 07.02 .2022$

\section{Bibliography \\ Pharmacopsychiatry 2022; 55: 255-265 \\ DOI 10.1055/a-1716-1856 \\ ISSN $0176-3679$}

(c) 2022. The Author(s).

This is an open access article published by Thieme under the terms of the Creative Commons Attribution-NonDerivative-NonCommercial-License, permitting copying and reproduction so long as the original work is given appropriate credit. Contents may not be used for commercial purpose, o adapted, remixed, transformed or built upon. (https://creativecommons. org/licenses/by-nc-nd/4.0/)

Georg Thieme Verlag KG, Rüdigerstraße 14,

70469 Stuttgart, Germany

\section{Correspondence}

Karin Egberts, MD

University Hospital of Wuerzburg, Centre for Mental Health

Department of Child and Adolescent Psychiatry,

Psychosomatics and Psychotherapy

Margarete-Höppel-Platz 1

D-97080

Wuerzburg

Germany

egberts_k@ukw.de

\section{ABSTRACT}

Introduction Despite the growing evidence base for psychotropic drug treatment in pediatric patients, knowledge about the benefit-risk ratio in clinical practice remains limited. The 'Therapeutic Drug Monitoring (TDM)-VIGIL' study aimed to evaluate serious adverse drug reactions (ADRs) in children and adolescents treated with antidepressants and/or antipsychotics in approved ('on-label'), and off-label use in clinical practice. Methods Psychiatric pediatric patients aged 6-18 years treated with antidepressants and/or antipsychotics either on-label or off-label were prospectively followed between October 2014 and December 2018 within a multicenter trial. Follow-up included standardized assessments of response, serious ADRs and therapeutic drug monitoring.

Results 710 youth (age $=14.6 \pm 2.2$ years, female $=66.6 \%$ ) were observed for 5.5 months on average; $76.3 \%$ received antidepressants, $47.5 \%$ antipsychotics, and $25.2 \%$ both. Altogether, $55.2 \%$ of the treatment episodes with antidepressants and $80.7 \%$ with antipsychotics were off-label. Serious ADRs occurred in $8.3 \%$ $(95 \% \mathrm{Cl}=6.4-10.6 \%)$ of patients, mainly being psychiatric adverse reactions ( $77.4 \%$ ), predominantly suicidal ideation and behavior. The risk of serious ADRs was not significantly different between patients using psychotropics off-label and on-label (antidepressants: $8.1 \%$ vs. $11.3 \%, p=0.16$; antipsychotics: $8.7 \%$ vs $7.5 \%, p=0.67)$. Serious ADRs occurred in $16.6 \%$ of patients who were suicidal at enrollment versus $5.6 \%$ of patients who were not suicidal (relative risk 3.0, $95 \% \mathrm{Cl}=1.9-4.9$ ).

Conclusion Off-label use of antidepressants and antipsychotics in youth was not a risk factor for the occurrence of serious ADRs in a closely monitored clinical setting. Results from large naturalistic trials like ours can contribute to bridging the gap between knowledge from randomized controlled trials and real-world clinical settings.

\section{Introduction}

Antidepressants and antipsychotics are widely prescribed, both onand off-label, to children and adolescents worldwide for a variety of indications such as mood disorders, anxiety disorders, behavioral disorders, or psychosis [1,2]. Although several meta-analyses contributed to a deeper understanding of their efficacy and safety [3-5] much uncertainty remains in the pediatric population in daily clinical practice [6,7]. At the moment of approval of a new drug, the knowledge about its benefit-risk ratio is far from complete since the pre-approval studies were usually carried out with a relatively small number of highly selected patients and with a short follow-up [8, 9]. Especially, information about serious and long-term adverse drug reactions (ADRs) is sparse [10]. Minors, as well as patients with intellectual disabilities, suicidal ideation, severe somatic comorbidities, or polypharmacy, are usually not included in clinical trials, but common in psychiatric practice [11], where the patients are not only much more heterogeneous $[11,12]$ but are often treated for longer periods and monitored less closely than in trials.

In addition, off-label prescribing is very common in child and adolescent psychiatry, with frequencies of around $50 \%$ for antide- pressants [13-15] and up to $95 \%$ for antipsychotics $[14,16]$ in European studies. This is concerning, as off-label prescribing exposes patients to unknown risks of ineffective or even harmful treatment $[3,17,18]$. Safety concerns may also lead to the delay, underuse, or rejection of potentially effective psychotropic drug treatments [3,4], which can negatively affect long-term results $[19,20]$. Moreover, psychotropic drugs are among the types of medication for which ADRs were most commonly reported in children and adolescents $[10,21]$. Treatment during a period of life when the patient undergoes marked developmental, hormonal, and neurobiological changes often requires dosing regimens different from adults and may result in burdensome and potentially severe ADRs, which in minors may differ in frequency, severity, and nature from those in adults [22]. For example, children treated with antipsychotics seem to have a greater risk of cardio-metabolic [23] and endocrine disturbances [24] like weight gain or type 2 diabetes mellitus [25], as well as neurological complications, e. g., somnolence, compared with adults [26, 27]. The perceived severity of ADRs and their impact on daily lives in pediatric patients may also differ from that in adults. The possibly increased risk of suicidal ide- 
ations and behaviors in childhood and adolescence when starting selective serotonin reuptake inhibitors (SSRIs) has received much attention and led to safety alerts by regulatory agencies, despite uncertainty about causality $[28,29]$.

Given these critical safety issues with potentially high impact on the individual patient and the resulting burden on health care systems, the importance of short- and long-term drug safety monitoring as well as the introduction of efficient pharmacovigilance methods in pediatric trials and as part of routine clinical practice have been increasingly recognized [21, 22, 27, 30, 31]. However, surveillance on ADRs is far from optimal, as appropriate monitoring practices are not yet established or were found to be inconsistent, and underreporting is still an unsolved problem [32, 33].

It is often assumed that the incidence and risk of ADRs are even higher in patients treated off-label than in individuals treated onlabel, as there are many potential problems associated with off-label treatment including the unavailability of appropriate pediatric drug formulations, poor prescribing information, and the risk of medication errors [34]. However, there is still a lack of clarity surrounding off-label use in the pediatric population due to the limited number of available studies, small samples, different methodologies and settings, varying definitions of ADRs, and off-label use in different studies [35], leading to controversial results [34, 3639]. However, all authors concluded that there is an urgent need for more research and stricter reporting and monitoring of ADRs for drugs prescribed off-label in children and adolescents [40].

In child and adolescent psychiatry, there are hardly any prospective pharmacovigilance studies, and the risks of ADRs associated with especially off-label prescribing of psychotropic drugs in minors are unclear [41]. Therefore, the objective of this prospective multicenter registry study was to evaluate suspected serious ADRs in children and adolescents treated with antidepressants and/or antipsychotics used in off-label or approved (on-label) use in daily clinical practice.

\section{Methods}

\section{Setting and study population}

This study was part of the therapeutic drug monitoring (TDM)-VIGIL study, which was funded by the German Federal Institute for Drugs and Medical Devices (BfArM-number V-15322/68605/20132018) and consisted of two subprojects. The overall goal of the first subproject was to investigate the (long-term) safety of antidepressants and antipsychotics in children and adolescents treated in daily clinical practice by a phase-IIIb/IV “large simple trial” (EudraCT 2013-004881-33) including standardized patient assessment and therapeutic drug monitoring (TDM). The second subproject aimed to evaluate the safety profile of psychostimulants and other drugs to treat attention-deficit/hyperactivity disorder. As these drugs are approved in children and adolescents, a prospective investigation was possible within an observational trial (BfArM-number 73.05/3832-397285/12).

This paper reports the design and main results of the first subproject. The study was conducted between October 2014 and December 2018 in 18 child and adolescent psychiatric centers in Germany, Austria, and Switzerland, forming the TDM-VIGIL consorti- um [42]. The consortium consisted of nine university departments, eight general hospital departments and a private specialist practice, all being members of the competence network for TDM in child and adolescent psychiatry (www.tdm-kjp.com), which has been described elsewhere [43]. All inpatients, day-unit patients, and outpatients aged 4-18 years for whom it was intended to start treatment with a new antidepressant or antipsychotic (including switchers) were eligible. Parents or legal guardians and, in the case of adolescents aged 14 and above, also the patients themselves had to provide informed consent for participation. The only exclusion criteria were an absolute contraindication to the drug and participation of the patient in another clinical trial. Recruitment of 1,000 patients was planned. The independent ethics committees of all participating centers approved the study, which was carried out in accordance with the principles of Good Clinical Practice and the Declaration of Helsinki.

\section{Study design and assessment of patient characteristics}

The study included five main visits during routine treatment: (i) baseline, i. e., before the first dose of the new medication, ii) at the target dose in a steady-state of the clinically chosen medication (i. e., after at least 5 half-lives of the last dose increase), iii) at discharge/end of outpatient treatment, iv) at follow-up two weeks after discharge (FU2-weeks), and at follow-up 6 months (FU6months) after the last steady-state visit. Additionally, a non-binding number of additional visits was permitted. At each visit, a standardized assessment of clinical response, ADRs, and drug serum concentration (except FU2-weeks) was performed ( $\triangleright$ Fig. 1).

At baseline, psychopathological and physical examinations were conducted, and basic demographic information (e. g., age, sex, intelligence level), nicotine and drug abuse as well as the presence of acute suicidality were collected clinically. The next visit took place when the (provisional) target dose and steady-state of the study medication were reached. Additional steady-state visits had to be performed after medication changes, in case of (serious) adverse events and at discharge. The number of steady-state visits, the time intervals between these, and thus, the follow-up period varied between patients depending on the clinical course. Psychiatric diagnoses were made clinically and coded according to the International Classification of Diseases (ICD-10) [44]. Safety parameters (vital signs, body size, body weight, BMI) and somatic diseases were collected at every visit ( $\triangleright$ Table 1 ). To document the severity and change of the patients' psychopathology the Clinical Global Impression subscales for severity and improvement (CGI-S and CGI-I) [45] were used. The Global Assessment of Functioning Scale (GAF) [46] was used to assess the everyday-effectiveness of the study medication. Drug adherence was assessed at each visit and objectified by TDM. Serum concentrations were determined according to the consensus guidelines for TDM in neuropsychopharmacology [47] by the TDM-laboratory of the University Hospital Wuerzburg, which offers a comprehensive TDM-service including specialist diagnostic interpretation and advice.

\section{Study medication}

Antidepressants and antipsychotics - defined according to the anatomical therapeutic chemical (ATC)-code N06A and N05A, exclud- 


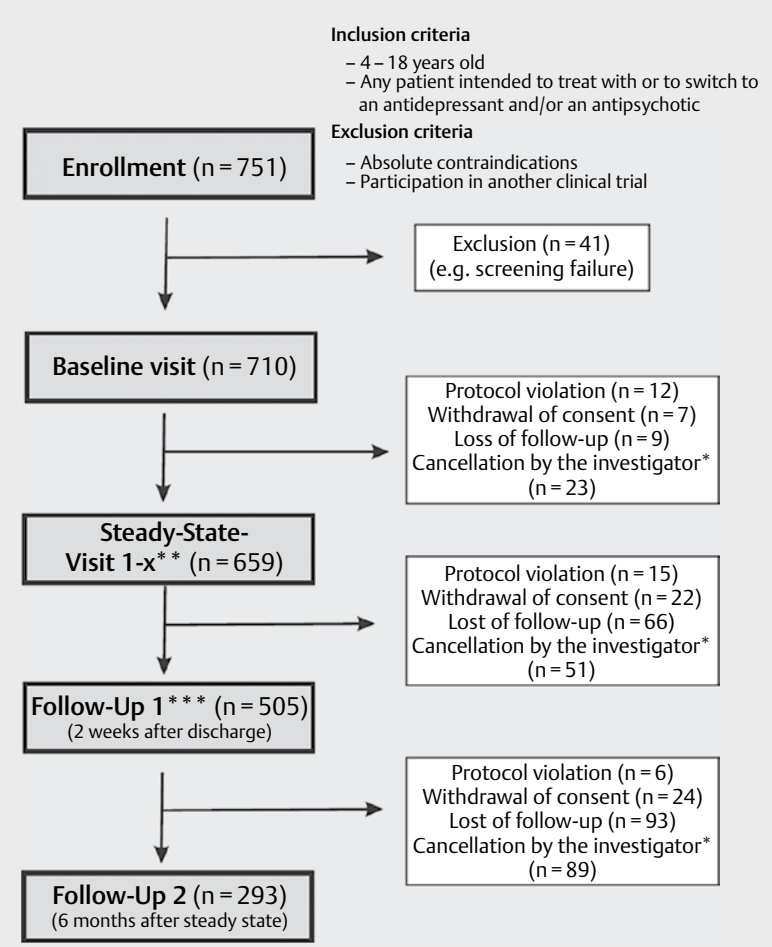

- Fig. 1 Patient disposition, visit plan, and course of study. * comprises cancellation due to non-response, ADRs, patient non-compliance and end of project; ${ }^{* *}$ Steady-State-visit 1 at target dose, followed by an optional number of steady state visits 2 -x according to the clinical needs and an obligatory visit at discharge (at the end of outpatient treatment episode, respectively); ${ }^{* * *} \ln 26$ patients no Follow-Up 1 but Follow-Up 2 was performed. ing lithium [WHO Collaborating Centre for Drug Statistics Methodology, www.whocc.no] - were prescribed upon clinician's choice either on- or off-label based on clinical judgment. The study protocol had no influence on the selection of the active substance, formulation, dosing regimen, and frequency, nor the duration of use. Both, combination therapy with different antidepressants and/or antipsychotics, as well as simultaneous treatment with other psychotropic (e. g., psychostimulants, benzodiazepines, mood-stabilizers) or somatic drugs, were permitted and prescription details were recorded in the medication log. Each treatment episode (i. e., administration of the same active substance at the same or different doses with a maximum of one-day interruption) of the study medication was classified as either on-label or off-label. Off-label treatment was defined as use either outside the approved age range, to treat symptoms or illnesses different from the approved ones, or use longer than the approved treatment duration (i. e., in case of risperidone that is only approved in Germany for 6 weeks in patients with intellectual disability). Off-label status was retrospectively evaluated according to the German regulatory authorization status (summary of product characteristics). Polypharmacy was defined as $\geq 7$ days of concomitant use of $\geq 2$ different psychotropic drugs.

\section{Assessment of ADRs and clinical response}

The Pediatric Adverse Event Rating Scale (PAERS), a semi-structured interview designed to assess any possible type of adverse events (AEs) in pediatric patients treated with psychotropic drugs, had to be administered at every visit [48]. The severity of the symptoms, functional impairment, and suspected connection with the medication were assessed for each AE. If an AE was judged as 'severe' or 'extremely severe' using PAERS, regardless of whether or not the

- Table 1 Assessment instruments and frequency

\begin{tabular}{|c|c|c|c|c|c|}
\hline & Baseline & $\begin{array}{l}\text { Steady State } \\
\text { visit 1-x }\end{array}$ & Discharge & FU2-weeks & FU6-months \\
\hline \multicolumn{6}{|l|}{ Standardized assessments } \\
\hline Patient characteristics (e. g., age, sex, setting, intelligence) & $\mathbf{x}$ & $\mathrm{x}$ & $\mathbf{x}$ & & $\mathbf{x}$ \\
\hline $\begin{array}{l}\text { Clinical parameters (e. g., weight, height, pulse, blood } \\
\text { pressure) }\end{array}$ & $\mathbf{x}$ & $\mathbf{x}$ & $\mathbf{x}$ & & $\mathbf{x}$ \\
\hline ICD10 diagnoses, target symptoms of medication & & $\mathbf{x}$ & $\mathbf{x}$ & & $\mathbf{x}$ \\
\hline TDM form (assessment of serum concentration) & $\mathbf{X}$ & $\mathbf{x}$ & $\mathbf{x}$ & & $\mathbf{x}$ \\
\hline Pediatric Adverse Event Rating Scale (PAERS) & $\mathbf{X}$ & $\mathbf{x}$ & $\mathbf{x}$ & $\mathbf{X}$ & $\mathbf{x}$ \\
\hline $\begin{array}{l}\text { Clinical Global Impression Scales Severity and } \\
\text { Improvement (CGI) }\end{array}$ & $\mathbf{x}$ & $\mathbf{x}$ & $\mathbf{x}$ & $\mathbf{x}$ & $\mathbf{X}$ \\
\hline Global Assessment Functioning Scale (GAF) & $\mathbf{x}$ & $\mathbf{x}$ & $\mathbf{x}$ & $\mathbf{x}$ & $\mathbf{X}$ \\
\hline diagnostic interview (by phone) & & & & $\mathbf{X}$ & \\
\hline \multicolumn{6}{|l|}{ Case documentation } \\
\hline Psychotropic medication protocol & $\mathbf{X}$ & $\mathbf{x}$ & $\mathbf{x}$ & $\mathbf{X}$ & $\mathbf{X}$ \\
\hline Somatic medication protocol & $\mathbf{x}$ & $\mathbf{X}$ & $\mathbf{X}$ & $\mathbf{X}$ & $\mathbf{X}$ \\
\hline \multicolumn{6}{|l|}{ End of study documentation form } \\
\hline \multicolumn{6}{|l|}{ Continuous safety assessment } \\
\hline $\mathrm{AE} / \mathrm{SAE}{ }^{*}$ screening form & $\mathbf{x}$ & $\mathbf{x}$ & $\mathbf{x}$ & $\mathbf{x}$ & $\mathbf{x}$ \\
\hline $\mathrm{AE} / \mathrm{SAE}$ reporting form & $\mathbf{x}$ & $\mathbf{x}$ & $\mathbf{x}$ & $\mathbf{X}$ & $\mathbf{x}$ \\
\hline $\mathrm{AE} / \mathrm{SAE}$ progress documentation form & $\mathbf{X}$ & $\mathbf{x}$ & $\mathbf{x}$ & $\mathbf{X}$ & $\mathbf{X}$ \\
\hline
\end{tabular}


symptom was judged as being related to the psychiatric medication, the study protocol requested the completion of a separate form for screening and documentation of serious adverse events (SAEs). An SAE was defined according to the definition in human drug trials (www.fda.gov/safety/medwatch/howtoreport/ucm053087. $\mathrm{htm}$ ) as an unfavorable event that results in death, is life-threatening, requires hospitalization or prolongation of an existing hospital stay, leads to persistent or significant disability, congenital anomaly, or another important medical condition. As soon as a SAE was recognized, clinicians had to fill out the SAE-reporting form according to the German medical agency (BfArM). SAEs were classified according to the Medical Dictionary for Regulatory Activities (MedDRA, version 22.0), and measures taken to address them as well as their outcome were documented. SAEs were reported to the supervisory authorities and ethics committees in accordance with the legal requirements of the respective country. The likelihood that the SAE was a serious ADR, i. e. that the suspected drug may have caused the adverse event, was assessed by the local investigator and finally validated by the study board using the algorithm of the WHO-UMC-Causality Assessment [https://cdn.who.int/ media/docs/default-source/medicines/pharmacovigilance/whocausality-assessment.pdf)]. SAEs assessed as 'probable' causality (i. e., event with reasonable time relationship to drug intake unlikely to be attributed to disease or other drugs) or 'possible' causality (i. e., event with reasonable time relationship to drug intake, although it could also be explained by disease or other drugs) were classified as serious ADRs. To record the impact of ADRs on the patients' overall functioning, the corresponding questions of the CGIefficacy index had to be answered for each study drug at each visit [71].

\section{Data management and statistical analysis}

An internet-based patient registry (secuTrial-system) was used for data acquisition, which met all legal requirements according to GCP, the European Medicines Agency, the US Food and Drug Administration, and the German Medicines Act. Data were entered directly into the electronic Case Report Forms by authorized staff, who were given access to the registry after special training. Data monitoring was carried out by the Center for Clinical Studies at the University Hospital Wuerzburg. The sample distributions of patients' characteristics, variables, and scores, which were recorded as quantitative data at ordinal, nominal, or ratio scales, were described by appropriate summary statistics as location parameters and measures of variation. Summary statistics were given stratified by age cohorts, sex, medication groups, and visits. Interval estimates for proportions of SAEs and serious ADRs were given as $95 \%$ confidence intervals $(95 \% \mathrm{Cl})$ using the Wilson score method. The primary endpoint was the proportion of patients who had at least one serious ADR during on- and off-label treatment. We compared distributions, proportions, and mean values between independent groups (Type III F-test, tests of the Wald-type) or between paired samples (Friedman-test, Wilcoxon-signed rank test). Categorical data were displayed in contingency tables and, where applicable, chi-square tests were used to examine whether there was an association between variables or groups. Statistical significance was defined as $\mathrm{p}<0.05$ (two-sided) without adjustments for multiple test- ing. Statistical analysis was carried out using the software IBM SPSSStatistics 25 and SAS 9.4.

\section{Results}

\section{Characteristics of the study population}

Altogether, 710 patients ( $66.6 \%$ girls, $77.5 \%$ inpatients) with a mean age of 14.6 (range 6-18) years were included; $8.9 \%$ were children younger than 12 years. The most common psychiatric diagnoses were depressive disorders in about $70 \%$ of the patients, followed by eating (22\%), behavioral (16\%), and anxiety (15\%) disorders. At enrollment, $25.5 \%$ were clinically judged to be suicidal. Relevant concomitant somatic diagnoses were reported in $13.2 \%$, especially broncho-pulmonary diseases, endocrine disorders, or digestive system diseases. $43.3 \%$ of the children and adolescents received psychotropic polypharmacy. Patient follow-up was on average 5.5 months (range $1-936$, median $=157.0$, interquartile range $=52.0-239.5$ days). The distribution of patients across the centers as well as the patients' baseline characteristics are summarized in $>$ Table 2.

\section{Study medication}

Seven hundred of the 710 in TDM-VIGIL included patients who had one or more treatment episodes with antidepressants and/or antipsychotics. For the remaining 10 patients, there was the intention to prescribe a new antidepressant or antipsychotic, but they did not receive it, and they were excluded from further analyses. Overall, 1,265 treatment episodes with study medication were registered with an average of 1.8 (range 1-14, median 1.0) episodes per patient. Among the patients, $76.3 \%$ received at least one antidepressant, $47.5 \%$ at least one antipsychotic, and $25.2 \%$ one or more substances from both drug classes. Fifteen different antidepressants, mainly SSRIs as well as noradrenergic and specific serotoninergic antidepressants (79.9\%) were used, most frequently fluoxetine (49.9\% of all antidepressants), sertraline (20.4\%), and mirtazapine (14.0\%). Among antipsychotics, mainly second-generation antipsychotics (SGAs, $80.5 \%$ ) were prescribed, most commonly aripiprazole ( $27.3 \%$ of all antipsychotics), quetiapine (21.0\%), olanzapine (18.8\%), and risperidone (9.4\%). Overall, $66.3 \%$ of the treatment episodes were classified as off-label $(55.2 \%$ involving antidepressants, $80.7 \%$ involving antipsychotics), most of these were due to age (63.2\%) and indication (35.0\%). More than two-thirds (69.7\%) of the patients received at least one antidepressant or antipsychotic under off-label conditions.

\section{Incidence of serious adverse events and (serious) adverse drug reactions}

ADRs were documented using PAERS across the five main visits in $57.7 \%$ of the 700 children and adolescents while they were treated with an antidepressant or antipsychotic. The total number of ADRs reported in these patients was 2,066 (i. e., on average 3 per patient), of which 5.3\% ADRs were judged as either severe or extremely severe. In patients with one or more antidepressant episodes, 983 ADRs were reported during antidepressant use, of which $5.3 \%$ ADRs were judged as (extremely) severe. In patients receiving only antipsychotics, 932 ADRs occurred, of which $4.2 \%$ were judged as 
- Table 2 Baseline characteristics of the study population $(n=710)$

\begin{tabular}{|c|c|}
\hline $\begin{array}{l}\text { Distribution of patients across centers } \\
\mathrm{n}(\%)\end{array}$ & \\
\hline Bad Wildungen & $57(8.0)$ \\
\hline Berlin I Charité & $41(5.8)$ \\
\hline Berlin II Vivantes & $8(1.1)$ \\
\hline Freiburg & $27(3.8)$ \\
\hline Fulda & $5(0.7)$ \\
\hline Heidelberg & $39(5.5)$ \\
\hline Cologne & $35(4.9)$ \\
\hline Mannheim & $10(1.4)$ \\
\hline Munich I LMU & $38(5.4)$ \\
\hline Munich II & $12(1.7)$ \\
\hline Neuruppin & $25(3.5)$ \\
\hline Regensburg & $18(2.5)$ \\
\hline Schweinfurt & $28(3.9)$ \\
\hline Tuebingen & $41(5.8)$ \\
\hline Ulm & $59(8.3)$ \\
\hline Vienna & $53(7.5)$ \\
\hline Wuerzburg & $165(23.2)$ \\
\hline Zuerich & $49(6.9)$ \\
\hline \multicolumn{2}{|l|}{ Sex $n(\%)$} \\
\hline Female & $473(66.6)$ \\
\hline Male & $237(33.4)$ \\
\hline Children < 12 years, $\mathrm{n}(\%)$ & $63(8.9)$ \\
\hline Adolescents $\geq 12$ years, $\mathrm{n}(\%)$ & $647(91.1)$ \\
\hline Age (years) mean $\pm S D$, range & $14.6 \pm 2.2,6-18$ \\
\hline Age girls (years) mean $\pm S D$ & $14.9 \pm 1.7$ \\
\hline Age boys (years) mean $\pm S D$ & $14.0 \pm 2.9$ \\
\hline \multicolumn{2}{|l|}{ Setting $\mathrm{n}(\%)$} \\
\hline Inpatient & $550(77.5)$ \\
\hline Day clinic patient & $104(14.6)$ \\
\hline Outpatient & $56(7.9)$ \\
\hline \multicolumn{2}{|l|}{ Anthropometric data (range) } \\
\hline Height (m) & $1.14-1.93$ \\
\hline Weight (kg) & $19.9-146.0$ \\
\hline BMI $\left(\mathrm{kg} / \mathrm{m}^{2}\right)$ & $10.8-43.6$ \\
\hline Patients with suicidality at baseline $n(\%)$ & $181(25.5)$ \\
\hline \multicolumn{2}{|l|}{ Intelligence level n (\%) } \\
\hline average & $643(90.6)$ \\
\hline sub-average & $58(8.2)$ \\
\hline unknown & $9(1.3)$ \\
\hline \multicolumn{2}{|l|}{$\begin{array}{l}\text { Most common ICD-diagnoses, } n \text { (\%) of multiple } \\
\text { entries }\end{array}$} \\
\hline F 32.1 moderate depressive episode & $296(41.7)$ \\
\hline F 50.0 anorexia nervosa & $113(15.9)$ \\
\hline F 32.2 severe depressive episode & $95(13.4)$ \\
\hline F 40.1 social phobias & $63(8.9)$ \\
\hline F 42.2 obsessive-compulsive disorder & $53(7.5)$ \\
\hline F 90.0 Attention-deficit/hyperactivity disorder & $49(7.0)$ \\
\hline F 90.1 hyperkinetic conduct disorder & $41(5.8)$ \\
\hline F 33.1 recurrent depressive disorder & $35(4.9)$ \\
\hline F 43.1 posttraumatic stress disorder & $32(4.5)$ \\
\hline F 20.0 paranoid schizophrenia & $29(4.1)$ \\
\hline
\end{tabular}

Table 2 Continued.

\begin{tabular}{|c|c|}
\hline $\begin{array}{l}\text { Distribution of patients across centers } \\
\text { n (\%) }\end{array}$ & \\
\hline \multicolumn{2}{|l|}{ The severity of illness (CGI-S) at baseline $n(\%)$} \\
\hline Not assessable & $19(2.7)$ \\
\hline Not at all ill & $0(0.0)$ \\
\hline Borderline mentally ill & $0(0.0)$ \\
\hline Mildly ill & $6(0.9)$ \\
\hline Moderately ill & $79(11.3)$ \\
\hline Markedly ill & $332(47.4)$ \\
\hline Severely ill & $236(33.7)$ \\
\hline Extremely ill & $29(4.1)$ \\
\hline $\begin{array}{l}\text { GAF-Score at baseline }(\mathbf{n}=\mathbf{7 0 3}) \text { mean } \pm S D \text {, } \\
\text { median, range; } n(\%)\end{array}$ & $\begin{array}{l}42.3 \pm 11.3,44 \\
1-81\end{array}$ \\
\hline $0-10$ : Constant danger to oneself and others & $4(0.6)$ \\
\hline 10-20: Danger to oneself and others & $17(2.4)$ \\
\hline 20-30: Impairment of in almost all areas & $78(11.1)$ \\
\hline 30-40: Severe impairment in several areas & $133(18.9)$ \\
\hline 40-50: Serious impairment & $241(34.3)$ \\
\hline 50-60: Moderate difficulties & $186(26.5)$ \\
\hline 60-70: Several slight impairments & $41(5.8)$ \\
\hline 70-80: Slight impairment of performance & $2(0.3)$ \\
\hline 80-90: Good performance & $1(0.1)$ \\
\hline \multicolumn{2}{|l|}{ Patients with multiple simultaneous use (n (\%) } \\
\hline all psychotropics & $342(48.2)$ \\
\hline antidepressants and antipsychotics & $305(43.0)$ \\
\hline \multicolumn{2}{|l|}{$\begin{array}{l}\text { Patients with psychiatric co-medication, n (\%) } \\
\text { (multiple) entries }\end{array}$} \\
\hline Tranquilizer & $70(10.0)$ \\
\hline Stimulants/atomoxetine/guanfacine & $87(12.3)$ \\
\hline Mood stabilizer, anticonvulsants & $18(2.6)$ \\
\hline Biperiden & $16(2.3)$ \\
\hline Melatonin & $14(2.0)$ \\
\hline Other & $10(1.4)$ \\
\hline $\begin{array}{l}\text { Observational time (days) mean }( \pm S D) \text {, range, } \\
\text { median }(I Q R)\end{array}$ & $\begin{array}{l}167.0 \pm 140.2 \\
1-946,157.0 \\
(52.0-239.5)\end{array}$ \\
\hline \multicolumn{2}{|c|}{$\begin{array}{l}\text { ICD: International Classification of diseases, CGI-S = Clinical Global } \\
\text { Impression Scale }- \text { Subscale Severity, } \mathrm{n}=\text { number, } \mathrm{SD}=\text { standard } \\
\text { deviation, GAF = Global Assessment Functioning Scale, IQR = Inter- } \\
\text { quartile range }\end{array}$} \\
\hline
\end{tabular}

(extremely) severe. In patients who used an antidepressant and an antipsychotic at the same time, 151 ADRs were registered of which $12.4 \%$ were judged as (extremely) severe. In 12 patients (1.7\%), ADRs were the reason for the premature termination of the study ( $\triangleright$ Fig. 1).

The total number of SAEs identified from all regular visits (PAERS), additional visits, and between regular visits by the SAE screening form was 115 . These SAEs were reported in 62 patients (8.7\%, 95\%Cl: $6.8-11.1 \%)$, of whom $48(77.4 \%)$ were female and four were children (6.5\%). Altogether, $80.9 \%(n=93)$ of SAEs were psychiatric events, $19.1 \%(n=22)$ somatic, and $14.8 \%(n=17)$ were recorded as life-threatening. The psychiatric events ( $n=75$ (80.6\%) out of 93) were predominantly thoughts of suicide, suicidal actions, 
or attempted suicide, reported in 46 patients, of which 28 patients (60.9\%) were classified as suicidal at enrollment. No SAE resulted in permanent damage or was fatal.

Causality with the study medication was assessed as possible ( $n=80,69.6 \%$ ) or probable $(n=13,11.3 \%$ ) in $93(80.9 \%$ ) of the 115 SAE, which accordingly were classified as serious ADRs. Serious ADRs were reported in 59 (8.3\%, $95 \% \mathrm{Cl}: 6.4-10.6 \%)$ children and adolescents, of which 40 patients $(5.6 \%, 95 \% \mathrm{Cl} 4.1-7.7 \%)$ were treated with an antipsychotic and/or antidepressant drug in off-label use. Thirty-four patients had one serious ADR, 18 patients had two, five patients had three, and two patients had four serious ADRs. None of them fulfilled the criteria of a suspected unexpected serious adverse reaction (SUSAR). Altogether, $68.8 \%$ of the serious $A D R s$ occurred during monotherapy with an antidepressant or antipsychotic. The distribution of serious ADRs by system-organclass and drug involved is shown in > Table 3.

The risk of serious ADRs was not significantly different between patients using psychotropics off-label and on-label, neither for antidepressants (8.1\% vs $11.3 \%, \mathrm{p}=0.16)$ nor antipsychotics $(8.7 \%$ vs $7.5 \%, p=0.67$ ) ( $>$ Fig. 2 ). Serious ADRs occurred in $16.6 \%$ of patients who were suicidal at enrollment versus $5.6 \%$ of patients who were not suicidal at enrollment (relative risk 3.0, $95 \% \mathrm{Cl}=1.9-4.9$ ).

As a consequence of a serious ADR the medication was not changed in $63.4 \%$, discontinued in $19.4 \%$ of the serious ADRs, the dose was reduced in $11.8 \%$, and increased in $3.2 \%$ of the cases. Among the serious ADRs, $36.6 \%$ required hospitalization or transfer of the patient to another ward, $3.2 \%$ the consultation of a medical specialist, and in $58.1 \%$ no general actions were taken (two events without specification). The majority of serious ADRs took a favorable course with symptoms completely subsiding in $84.9 \%$ and partially subsiding in $5.4 \%$, while $7.5 \%$ were unchanged, and the outcome was not reported for two (2.2\%) cases at the end of the observational period.

\section{Discussion}

The results from our prospective pharmacovigilance study show that on average $8.3 \%$ of children and adolescents treated in daily clinical practice with various antidepressant and/or antipsychotic drugs suffered from suspected serious ADRs. The incidence of serious ADRs in patients using antidepressants and/or antipsychotics off-label was not different from those using the study medication on-label. The most frequent serious ADRs were suicide attempts. Serious ADRs occurred three times more often in patients with suicidality at enrollment.

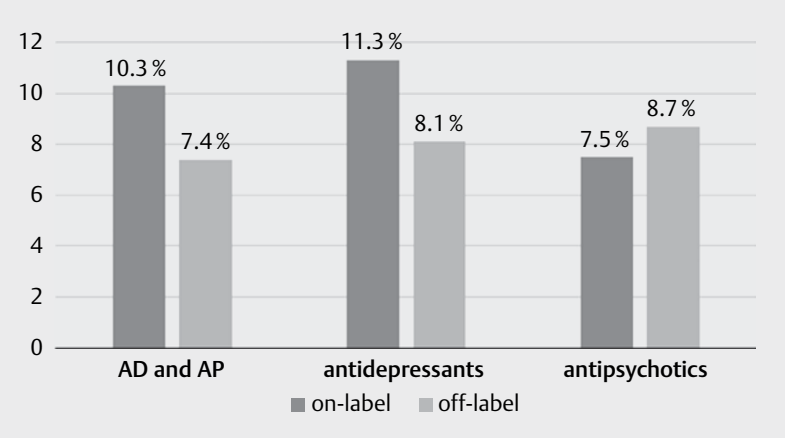

- Fig. 2 Serious adverse drug reactions (ADRs) per episode of medication in on-label and off-label use. The percentage of serious ADRs is shown in relation to the total number of medication episodes with an antidepressant or/and an antipsychotic (left bars), an antidepressant (middle bars) and an antipsychotic (right bars) reported in the study. The numbers do not add up to the total number because antidepressants (AD) and/or antipsychotics (AP) in on-label and off-label use could be involved in the same event. Such serious ADRs could contribute to the event rates of AD as well as AP, but only once to the event rate of all episodes (AD plus AP).

- Table 3 Distribution of serious adverse drug reactions by system organ class and drug involved

\begin{tabular}{|c|c|c|c|c|c|c|}
\hline SOC & Total n (\%) ${ }^{*}$ & $\begin{array}{l}\text { Fluoxetine n } \\
\text { (\%) }\end{array}$ & $\begin{array}{l}\text { Aripiprazole n } \\
\text { (\%) }\end{array}$ & $\begin{array}{l}\text { Sertraline } n \\
\text { (\%) }\end{array}$ & $\begin{array}{l}\text { Quetiapine n } \\
\text { (\%) }\end{array}$ & $\begin{array}{l}\text { Other drugs n } \\
\text { (\%) }\end{array}$ \\
\hline Psychiatric disorders & $72(77.4)$ & $36(85.7)$ & $13(65.0)$ & $8(80.0)$ & $12(92.3)$ & $38(71.7)$ \\
\hline ,Suicidality“ & $62(66.7)$ & $32(75.2)$ & $10(50.0)$ & $6(80.0)$ & $11(84.7)$ & $25(47.1)$ \\
\hline Suicide attempts & $29(31.2)$ & $12(28.6)$ & $6(30.0)$ & $0(0.0)$ & $5(38.5)$ & $20(37.7)$ \\
\hline Suicidal ideation & $27(29.0)$ & $17(40.0)$ & $3(15.0)$ & $2(20.0)$ & $4(30.8)$ & $5(9.4)$ \\
\hline Suicidal behaviour & $6(6.5)$ & $3(7.1)$ & $1(5.0)$ & $6(60.0)$ & $2(15.4)$ & $0(0.0)$ \\
\hline other psychiatric disorders & $10(10.8)$ & $4(9.5)$ & $3(15.0)$ & $0(0.0)$ & $1(7.7)$ & $13(24.5)$ \\
\hline Nervous system & $11(11.8)$ & $2(4.8)$ & $4(20.0)$ & $0(0.0)$ & $1(7.7)$ & $7(13.2)$ \\
\hline Investigations & $3(3.2)$ & $1(2.4)$ & $2(10.0)$ & $1(10.0)$ & $0(0.0)$ & $0(0.0)$ \\
\hline Blood lymphatic system & $3(3.2)$ & $1(2.4)$ & $1(5.0)$ & $0(0.0)$ & $0(0.0)$ & $2(3.8)$ \\
\hline Gastrointestinal & $2(2.2)$ & $1(2.4)$ & $0(0.0)$ & $1(10.0)$ & $0(0.0)$ & $0(0.0)$ \\
\hline Cardiac disorders & $1(1.1)$ & $0(0.0)$ & $0(0.0)$ & $0(0.0)$ & $0(0.0)$ & $1(1.9)$ \\
\hline Endocrine disorders & $1(1.1)$ & $1(2.4)$ & $0(0.0)$ & $0(0.0)$ & $0(0.0)$ & $1(1.9)$ \\
\hline Total $^{* *}$ & 93 & 42 & 20 & 10 & 13 & 53 \\
\hline
\end{tabular}


Our results are somewhat consistent with those in prior studies. For example, a naturalistic study in 184 pediatric outpatients treated with SGAs reported at least one ADR in about $71 \%$ of patients; $9 \%$ of ADRs, observed in $6.4 \%$ of the patients, were classified as serious. Like in our study, ADRs were also mainly psychiatric events, which the authors considered as partial or total lack of response [49]. Another prospective study on the safety of antipsychotics in children and adolescents classified $10.1 \%$ of the ADRs recorded under naturalistic conditions as serious, which were observed in $5.7 \%$ of patients, and of which one third were psychiatric effects [75]. These authors point out, that concerning psychiatric ADRs, it is difficult to assign causality to psychotropic drugs with high reliability because of a potential worsening of the underlying condition and thus insufficient treatment effects [50]. The most common 'adverse event' in our study - 'suicidality' - is at the same time a typical symptom of depression, which was the most frequent diagnosis in the sample and is known to be a strong risk factor for suicide [51]. Our finding illustrates the complex relationship between the reason to prescribe a drug, the intended drug action, and (potential) ADR. In our study, it was therefore extremely difficult to unravel whether suicidality as an adverse event was caused by the drug (i. e., was an ADR) or was an insufficient response to the drug or was an event in the natural course of the disease. This is underlined by the fact that the causality with the medication for almost all psychiatric (in contrast to somatic) serious ADRs in our study was classified as 'possible' (not as 'probable'). This causality dilemma, that the adverse effects of antidepressant drugs may mimic the symptoms of the underlying disease [33], is well known and also reflected in the debate on suicidality associated with the use of SSRIs in youth [28, 29].

More than a quarter of patients included in our study were judged to be suicidal at enrollment underlining that suicidality is an important and increasing reason for seeking treatment. However, suicidal patients are usually excluded from clinical trials as well as patients who are treated with more than one psychoactive drug at the same time. The fact that concomitant drug use is considered as a risk factor for ADRs $[52,53]$ is supported by our finding that the proportion of (extremely) severe ADRs assessed by PEARS was higher in patients using antidepressants and antipsychotics at the same time. However, any comparison between the incidence and prevalence rates of ADRs reported in clinical trials is difficult to draw, as there are large differences in the classification systems, recorded periods, treatment settings, methods of data assessment, and patient and medication groups. Additionally, information about the severity of the ADRs is often missing [31].

The proportion of off-label prescriptions in our population was slightly higher than in previous epidemiological studies from Germany in antidepressants (40.9-49.1\%) [13, 15] and antipsychotics (52.3-71.1\%)[16]. This could be explained by the fact that the majority of our patients were inpatients in specialized centers and that specialist treatment by hospital doctors increases the likelihood of receiving an off-label prescription [13]. We did not find that off-label prescribing was associated with a more frequent occurrence of serious ADRs than on-label prescribing. Our data, therefore, suggest that off-label use of antidepressants and antipsychotics in predominantly adolescents carries a lower risk of serious ADRs than off-label use in other, possibly more vulnerable pediatric popula- tions, such as infants [7,54], or off-label use of other drug classes, e. g., oncological drugs $[37,55]$. It should be mentioned that in our on- and off-label samples mostly identical drug classes (SSRIs, SGAs), sometimes even identical drugs (e. g., fluoxetine, sertraline, aripiprazole) were administered. The latter was the case when a drug, that is approved for children and adolescents in a single specific indication (e. g., major depression), was used for other indications (e. g. anxiety disorders). Although it is often stated that offlabel prescribing leads to more frequent ADRs, the evidence for this statement is scarce, specifically for off-label prescription of psychotropic drugs in minors. A study that analyzed spontaneously reported psychiatric ADRs to the Swedish Drug Information System database after treatment with any suspected drug class in children and adolescents during a 10-year period showed that serious ADRs were reported more frequently after off-label drug use than after drug use as labeled (25 (21.4\%) vs. 10 (7.5\%) individual case safety reports; $p=0.002)$. However, this study could not provide incidence estimates due to the limitations of spontaneous reporting systems [41]. A large epidemiological study based on health insurance data of around two million German children and adolescents between 2004 and 2011, found that, similar to our findings, incidence rates (IR) (per 10,000 Person-Years) of adverse events were not significantly different between on- and off-label use, e. g. poisoning by antipsychotics (on-label users $I R=7.8 \mathrm{vs}$. off-label users $I R=9.2$ ) and extrapyramidal events (on-label $I R=3.7$ vs off-label $I R=1.39$ ) [22], as well as cardio-/cerebrovascular (on-label $I R=9.8$ vs. off-label $I R=4.5$ ), and suicidal events (on-label $I R=35.2$ vs. offlabel $I R=21.7$ ) with antidepressants [15]. A possible explanation for our findings could be that the standardized safety measures including TDM with specialist diagnostic interpretation and recommendation - carried out as part of the study could have had a preventive effect on the development of serious ADRs under off-label conditions and could also have contributed to their most favorable course. In our study, a higher proportion of serious ADRs had a positive outcome than in the naturalistic studies cited above, in which (mostly non-serious) ADRs observed in youth during antipsychotic pharmacotherapy fully recovered in $27 \%$ and improved in $24 \%$ of cases [49], or fully recovered in $17.6 \%$ and improved in $38.2 \%$ of cases [50]. Unfortunately, no separate information about the outcome of serious ADRs was provided in either of these studies.

TDM in children and adolescents is a helpful tool in daily clinical practice to monitor psychopharmacotherapy $[30,47,56]$ and might be even more relevant when antidepressants and antipsychotics are used off-label because although the risk of serious ADRs might not be higher, the effectiveness and thus the benefit-risk balance can be different in off-label use. Considering the precarious approval situation for psychotropics in minors in Germany [57] and many countries, also the question arises whether collecting and analyzing standardized TDM-data could be used to generate safety data, which is important since the off-label status will probably not change in this population for the foreseeable future.

\section{Limitations}

The findings of the present study must be interpreted in the context of several limitations. First, although we were able to investigate 700 patients treated with the study medication, our sample size was too small and too heterogeneous to evaluate and compare 
the occurrence of serious ADRs for individual antidepressants and antipsychotics, or specific diagnoses or the younger age group. Second, the medication choice, starting dose, titration speed, and target dose, which are all related to the risk of serious ADRs, were naturalistically chosen based on the clinician's judgment and varied greatly. Third, our study was conducted in patients who were treated at specialized child and adolescent psychiatric centers and who were closely and regularly monitored, assessed, and evaluated. Therefore, the generalizability of our findings to primary care settings or settings without intensive monitoring in our study might be limited. Fourth, there are large differences between countries in the approval status of psychotropic drugs for pediatric patients with a comparatively restrictive approval policy in Germany. Finally and most importantly, causality assessment is difficult in psychiatric patients with often complex clinical conditions who receive often multiple psychotropic drugs and in whom the ADRs could also be symptoms of the underlying disease. This may have led to an over- or underestimation of the incidence of serious ADRs. However, we believe that this is not systematically different between on- and off-label use.

\section{Strengths}

One strength of our study is that 'real-world data' were generated by 18 centers prospectively and in a standardized way and assuring the data quality by controlled training and data-monitoring procedures. 'Real world' patient samples derived from healthcare databases are usually not characterized as completely and precisely as our sample, and also safety data in observational trials usually are poorly or inconsistently reported [6]. In our naturalistic sample, the ADR classification of clinical trials was used, and with the use of (self-report) rating scales and interviews, a comprehensive capturing of adverse events was assured. We used an internetbased patient registry to perform a multicenter trial, involving hospitals in three European countries. The chosen method of the prospective standardized patient and drug monitoring has proven to be a valuable approach to post-marketing surveillance in daily patient care. In addition, we included patients as heterogeneous as they are in clinical practice without applying the exclusion criteria that are common in clinical trials, such as suicidality, comorbidities, and cotreatments. Therefore, we think that our sample is representative of the pediatric psychiatric population of specialized child and adolescent psychiatric centers, particularly of adolescents.

\section{Conclusions}

Off-label use of antidepressants and antipsychotics by children and adolescents was not a risk factor for serious ADRs in a routine clinical setting in which patients were closely monitored. However, the reported numbers of (serious) ADRs underline the need to further investigate the risk factors for their occurrence and the methods to prevent them in the pediatric population. Data derived from clinical practice like ours better reflect the reality of treatment and can contribute to bridging the gap between information available from randomized controlled trials and the real clinical setting [58] to support clinical decision making and guideline development and ulti- mately improve outcomes of youth receiving psychotropic medication treatment.

\section{Author contributions}

KE and MG wrote the article; MG, KE, MR, PH, and PP designed the research; KE, RT, SR performed the research; SU, MS, RT, SF, and SR performed and judged the laboratory measurements; UM and KE analyzed the data, GA and HR managed the patient registry and processed the data; $C C$ helped with the preparation of the manuscript; WB, CC, CF, AH, TH, HI, MKA, AK, MKO, KR, TR, SR, CR, GS, FT, CW, SW, and MR recruited patients, all authors reviewed the manuscript.

\section{Acknowledgements}

We gratefully acknowledge Andrea Bäuerle for her support in coordinating the study, Professor Jürgen Deckert and the TDM laboratory team of the Center for Mental Health of the University Hospital Wuerzburg for performing the concentration analyses, the staff of the Central Office for Clinical Studies at the University Hospital Würzburg for their advice in conducting the clinical trial, in particular Olga Miljukov for her support with the statistical analysis, Professor Toine Egberts, University Hospital Utrecht, for his scientific advice and the members of the Central Information Office Marburg $\mathrm{GmbH}$ for the development of the patient registry and the data management. We especially thank all local study staff and research assistants in the study centers and most of all the participating patients.

\section{Conflict of Interest}

KE, RT, MR, MG, and PP received grant research support from BfArM. MR currently receives a research grant from Kids-Safe, Innovation Committee of the German Federal Joint Committee (G-BA grant number 01NVF16021). AH received conference support, speaker's fees, and/or served in an advisory role for Shire/Takeda and Lilly; he was involved as an investigator in clinical trials by Shire, Takeda, Janssen-Cilag, Otsuka, Sunovion, Servier, Lundbeck, Nuvelution, Gedeon Richter, and Emalex. PH reports grants from the German Ministry of Research and Education, European Union, Berlin Chamber of Physicians, German Parkinson Society, University Hospital Wuerzburg, Robert Koch Institute, German Heart Foundation, Federal Joint Committee (G-BA) within the Innovationfond, German Research Foundation, Bavarian State (ministry for science and the arts), German Cancer Aid, grants from Charité - Universitätsmedizin Berlin (within Mondafis, Mondafis is supported by an unrestricted research grant to the Charité from Bayer), University Göttingen (within FIND-AF randomized; FIND-AF randomized is supported by an unrestricted research grant to the University Göttingen from Boehringer-Ingelheim), University Hospital Heidelberg (within RASUNOA-prime; RASUNOA-prime is supported by an unrestricted research grant to the University Hospital Heidelberg from Bayer, BMS, Boehringer-Ingelheim, Daiichi Sankyo. PP receives grant research support from the German Federal Ministry of Education and Research (BMBF) and was involved in clinical trials from Servier and Lundbeck; he received an advisor honorarium from Boehringer Ingelheim and speaker's honoraria from Shire, Infectopharm, and Gerot Lannach. CC has been a consultant and/or advisor to or has received honoraria from AbbVie, Acadia, Alkermes, Allergan, Angelini, Aristo, Axsome, Damitsa, Gedeon 
Richter, Hikma, IntraCellular Therapies, Janssen/J\&], Karuna, LB Pharma, Lundbeck, MedAvante-ProPhase, MedInCell, Medscape, Merck, Mitsubishi Tanabe Pharma, Mylan, Neurocrine, Noven, Otsuka, Pfizer, Recordati, Rovi, Servier, SK Life Science, Sumitomo Dainippon, Sunovion, Supernus, Takeda, Teva, and Viatris. He provided expert testimony for Janssen and Otsuka. He served on a Data Safety Monitoring Board for Lundbeck, Rovi, Supernus, and Teva. He has received grant support from Janssen and Takeda. He received royalties from UpToDate and is also a stock option holder of LB Pharma. SW has received in the last 5 years royalties from Thieme, Hogrefe, Kohlhammer, Springer, and Beltz. Her work was supported in the last 5 years by the Swiss National Science Foundation, diff. EU FP7s programs, Hochspezialisierte Medizin of the Kanton Zurich, Switzerland, BfArM, ZlnEP, Hartmann Müller Stiftung, Olga Mayenfisch, Gertrud Thalmann, Vontobel, Unicentia, Erika Schwarz Fonds, Gesundheitsförderung Schweiz. The other authors (GA, WB, SF, CF, TH, HI, UM, MKE, AK, MKO, KR, TR, HR, SR, CR, MS, GS, FT, SU, and CW) declare no (further) conflict of interest.

\section{References}

[1] Piovani D, Clavenna A, Bonati M. Prescription prevalence of psychotropic drugs in children and adolescents: An analysis of international data. Eur J Clin Pharmacol 2019; 75: 1333-1346

[2] Sultan RS, Correll CU, Schoenbaum M et al. National patterns of commonly prescribed psychotropic medications to young People. J Child Adolesc Psychopharmacol 2018; 28: 158-165

[3] Sharma AN, Arango C, Coghill D et al. BAP position statement: Off-label prescribing of psychotropic medication to children and adolescents. J Psychopharmacol 2016; 30: 416-421

[4] Solmi M, Fornaro M, Ostinelli EG et al. Safety of 80 antidepressants, antipsychotics, anti-attention-deficit/hyperactivity medications and mood stabilizers in children and adolescents with psychiatric disorders: A large scale systematic meta-review of 78 adverse effects. World Psychiatry 2020; 19: 214-232

[5] Correll CU, Cortese S, Croatto G et al. Efficacy and acceptability of pharmacological, psychosocial, and brain stimulation interventions in children and adolescents with mental disorders: An umbrella review. World Psychiatry 2021; 20: 244-275

[6] Persico AM, Arango C, Buitelaar JK et al. European Child and Adolescent Clinical Psychopharmacology Network. Unmet needs in paediatric psychopharmacology: Present scenario and future perspectives. Eur Neuropsychopharmacol 2015; 25: 1513-1531

[7] Aagaard L, Hansen EH. Adverse drug reactions from psychotropic medicines in the paediatric population: Analysis of reports to the Danish Medicines Agency over a decade. BMC Res Notes 2010; 3: 176

[8] Vitiello B, Correll C, van Zwieten-Boot B et al. Antipsychotics in children and adolescents: Increasing use, evidence for efficacy and safety concerns. Eur Neuropsychopharmacol 2009; 19: 629-635

[9] Zimmerman KO, Smith PB, McMahon AW et al. Duration of pediatric clinical trials submitted to the US Food and Drug Administration. JAMA Pediatr 2019; 173: 60-67

[10] Aagaard L, Christensen A, Hansen EH. Information about adverse drug reactions reported in children: A qualitative review of empirical studies. Br J Clin Pharmacol 2010; 70: 481-491

[11] Díaz-Caneja CM, Espliego A, Parellada M et al. Polypharmacy with antidepressants in children and adolescents. Int J Neuropsychopharmacol 2014; 17: 1063-1082

[12] Zimmerman M, Mattia JI, Posternak MA. Are subjects in pharmacological treatment trials of depression representative of patients in routine clinical practice? Am J Psychiatry 2002; 159: 469-473
[13] Dörks M, Langner I, Dittmann U et al. Antidepressant drug use and off-label-prescribing in children and adolescents in Germany: Results from a large population-based cohort study. Eur Child Adolesc Psychiatry 2013; 22: 511-518

[14] Braüner JV, Johansen LM, Roesbjerg T et al. Off-label-prescription of psychopharmacological drugs in child and adolescent psychiatry. J Clin Psychopharmacol 2016; 36: 500-507

[15] Schröder C, Dörks M, Kollhorst B et al. Extent and risks of antidepressant off-label use in children and adolescents in Germany between 2004 and 2011. Pharmacoepidemiol Drug Saf 2017; 26: 1395-1402

[16] Schröder C, Dörks M, Kollhorst B et al. Extend and Risk of Antipsychotic Off-Label-Use in Children and Adolescents in Germany between 2004 and 2011. J Child and Adolesc Psychopharmacol 2017; 27: 806-813

[17] Zito JM, Derivan AT, Kratochvil C] et al. Off-label psychopharmacologic prescribing for children: History supports close clinical monitoring. Child and Adolescent Psychiatry and Mental Health 2008; 2: 24

[18] Gerlach M, Greenhill L, Warnke A. Special features of psychopharmacological therapy in children and adolescents. Gerlach M, Warnke A, Greenhill L, eds. Psychiatric Drugs in Children and Adolescents. Basic Pharmacology and Practical Applications. Wien: Springer Verlag; 2014: 61-75

[19] Rubio JM, Correll CU. Duration and relevance of untreated psychiatric disorders, 1: Psychotic disorders. J Clin Psychiatry 2017; (a) 78: 358-359

[20] Rubio JM, Correll CU. Duration and relevance of untreated psychiatric disorders, 2: Nonpsychotic psychiatric disorders and substance use disorders. J Clin Psychiatry 2017; (b) 78: 464-465

[21] Carnovale C, Brusadelli T, Zuccotti G et al. The importance of monitoring adverse drug reactions in pediatric patients: The results of a national surveillance program in Italy. Expert Opin Drug Saf 2014; 13: $1-8$

[22] Gerlach M, Egberts K, Dang SY et al. Therapeutic drug monitoring as a measure of proactive pharmacovigilance in child and adolescent psychiatry. Expert Opin Drug Saf 2016; 15: 1477-1482

[23] Minjon L, van den Ban E, de Jong E et al. Reported adverse drug reactions in children and adolescents treated with antipsychotics. J Child Adolesc Psychopharmacol 2019; 29: 124-132

[24] Correll CU, Carlson HE. Endocrine and metabolic adverse effects of psychotropic medications in children and adolescents. J Am Acad Child Adolesc Psychiatry 2006; 45: 771-791

[25] Galling B, Roldán A, Nielsen RE et al. Type 2 diabetes mellitus in youth exposed to antipsychotics: A systematic review and meta-analysis. JAMA Psychiatry 2016; 73: 247-259

[26] De Hert M, Dobbelaere M, Sheridan EM et al. Metabolic and endocrine adverse effects of second-generation antipsychotics in children and adolescents: A systematic review of randomized, placebo controlled trials and guidelines for clinical practice. Eur Psychiatry 2011; 26: 144-158

[27] Pringsheim T, Panagiotopoulos C, Davidson J et al. CAMESA guideline group Evidence-based recommendations for monitoring safety of second generation antipsychotics in children and youth. J Can Acad Child Adolesc Psychiatry 2011; 20: 218-233

[28] Fornaro M, Anastasia A, Valchera A et al. The FDA "Black Box" Warning on Antidepressant Suicide Risk in Young Adults: More Harm Than Benefits? Front. Psychiatry 2019; 10: 294

[29] Dragioti E, Solmi M, Favaro A et al. Association of antidepressant use with adverse health outcomes: A systematic umbrella review. JAMA Psychiatry 2019; 76: 1241-1255

[30] Egberts K, Karwautz A, Plener PL et al. Pharmacovigilance in child and adolescent psychiatry. Z Kinder Jugendpsychiatr Psychother 2015; 43: 21-28 
[31] Coates M, Spanos M, Parmar P et al. A review of methods for monitoring adverse events in pediatric psychopharmacology clinical trials. Drug Saf 2018; 41: 465-471

[32] Rodday AM, Parsons SK, Mankiw C et al. Child and adolescent psychiatrists' reported monitoring behaviors for second-generation antipsychotics. J Child Adolesc Psychopharmacol 2015; 25: 351-361

[33] Carleton BC, Smith MA, Gelin MN et al. Paediatric adverse drug reaction reporting: Understanding and future directions. Can J Clin Pharmacol 2007; 14: e45-e57

[34] Nguyen KA, Mimouni Y, Jaberi E et al. Relationship between adverse drug reactions and unlicensed/off-label drug use in hospitalized children (EREMI): A study protocol. Therapie 2021; 76: 675-685

[35] Mason J, Pirmohamed M, Nunn T. Off-label and unlicensed medicine use and adverse drug reactions in children: a narrative review of the literature. Eur J Clin Pharmacol 2012; 68: 21-28

[36] Palmaro A, Bissuel R, Renaud N et al. Off-label prescribing in pediatric outpatients. Pediatrics 2015; 135: 49-58

[37] Aagaard L, Hansen EH. Prescribing of medicines in the Danish paediatric population out with the licensed age group: characteristics of adverse drug reactions. $\mathrm{Br}$ J Clin Pharmacol 2011; 71: 751-757

[38] Pratico AD, Longo L, Mansueto $S$ et al. Off-label use of drugs and adverse drug reactions in pediatric units: A prospective, multicenter study. Curr Drug Saf 2018; 13: 200-207

[39] Neubert A, Dormann H, Weiss J et al. The impact of unlicensed and off-label drug use on adverse drug reactions in paediatric patients. Drug Saf 2004; 27: 1059-1067

[40] Gore R, Chugh PK, Tripathi CD et al. Pediatric off-label and unlicensed drug use and its implications. Curr Clin Pharmacol 2017; 12: 18-25

[41] Bygdell M, Brunlöf G, Wallerstedt SM et al. Psychiatric adverse drug reactions reported during a 10 -year period in the Swedish pediatric population. Pharmacoepidemiol Drug Saf 2012; 21: 79-86

[42] Egberts K, Plener P, Malzhan U et al. Sicherheit von psychopharmaka bei kindern und jugendlichen in der klinischen praxis - Erkenntnisse einer prospektiven studie. Bulletin zur Arzneimittelsicherheit 2020; 3: 4-10

[43] Egberts KM, Mehler-Wex C, Gerlach M. Therapeutic drug monitoring in child and adolescent psychiatry. Pharmacopsychiatry 2011; 44: 249-253

[44] Remschmidt H, Schmidt M, Poustka F. eds. Multiaxiales Klassifikationsschema für psychische Störungen des Kinder- und Jugendalters nach ICD-10 der WHO. Bern: Huber Verlag; 2012

[45] Guy W. Clinical Global Impressions. ECDEU Assessment Manual for Psychopharmacology - Revised. Rockville, MD: U.S. Department of Health, Education, and Welfare; Psychopharmacology Research Branch. Division of Extramural Research Programs. 1976: 218-222
[46] Sass H, Wittchen H, Zaudig M et al eds. Diagnostische kriterien des diagnostischen und statistischen manuals psychischer störungen. DSM-IV-TR. Hogrefe Verlag; Göttingen: 2003: 47-49

[47] Hiemke C, Bergemann N, Clement HW et al. Consensus guidelines for therapeutic drug monitoring in neuropsychopharmacology: Update 2017. Pharmacopsychiatry 2018; 51: 9-62

[48] March J, Karayal O, Chrisman A. CAPTN: The pediatric adverse event rating scale. Novins DK, DeYoung, eds. The Scientific Proceedings of the 2007 Annual Meeting of the American Academy of Child and Adolescent Psychiatry. Boston; 2007: 241

[49] Rafaniello C, Pozzi M, Pisano S et al. Second generation antipsychotics in 'Real-life' paediatric patients. Adverse drug reactions and clinical outcomes of drug switch. Expert Opin Drug Saf 2016; 15: 1-8

[50] Cicala G, Barbieri MA, Santoro V et al. Safety and Tolerability of Antipsychotic Drugs in Pediatric Patients: Data From a 1-Year Naturalistic Study. Front. Psychiatry 2020; 11: 152

[51] Schneeweiss S, Patrick AR, Solomon DH et al. Comparative safety of antidepressant agents for children and adolescents regarding suicidal acts. Pediatrics 2010; 125: 876-888

[52] Hilt RJ, Chaudhari M, Bell JF et al. Side effects from use of one or more psychiatric medications in a population-based sample of children and adolescents. J Child Adolesc Psychopharmacol 2014; 24: 83-89

[53] Rashed AN, Wong IC, Cranswick N et al. Risk factors associated with adverse drug reactions in hospitalised children: International multicentre study. Eur J Clin Pharmacol 2012; 68: 801-810

[54] Kurian J, Mathew J, Sowjanya K et al. Adverse drug reactions in hospitalized pediatric patients: A prospective observational study. Indian J Pediatr 2016; 83: 414-419

[55] Nor Aripin KN, Choonara I, Sammons HM. Systematic review of safety in paediatric drug trials published in 2007. Eur J Clin Pharmacol 2012; 68: 189-194

[56] Kloosterboer SM, Vierhout D, Stojanova J et al. Psychotropic drug concentrations and clinical outcomes in children and adolescents: A systematic review. Expert Opin Drug Saf 2020; 19: 873-890

[57] Grau K, Fegert J, Plener PL. Psychopharmakologie in der KJP in Deutschland. Off-label-use und zulassung verfügbarer Fertigarzneimittel. Psychopharmakotherapie 2015; 22: 240-249

[58] Trifirò G, Gini R, Barone-Adesi F et al. The role of European healthcare databases for post-marketing drug effectiveness, safety and value evaluation: Where does Italy stand? Drug Saf 2019; 42: 347-363 